\title{
INFLUÊNCIA DA ATIVIDADE FÍ́SICA PROGRAMADA NA PRESSÃO ARTERIAL DE IDOSOS HIPERTENSOS SOB TRATAMENTO NÃO-FARMACOLÓGICO
}

Weimar Kunz Sebba Barroso*, Paulo César Brandão Veiga Jardim, Priscila Valverde Vitorino, Amanda Bittencourt, flávia Miquetichuc Trabalho realizado pela Liga de Hipertensão Arterial do Hospital das Clínicas da Faculdade de Medicina da Universidade Federal de Goiás, Goiânia, GO

* Correspondência Rua T-48, número 100, Apt 500 - Setor Oeste CEP 74140130 Goiânia - GO

\begin{abstract}
RESUMO
Oвjetivo. Avaliar a influência da atividade física na pressão arterial de pacientes idosos hipertensos sob tratamento nãofarmacológico (TNF).

Métodos. Estudamos homens e mulheres, maiores de 60 anos, com hipertensão arterial estágio I, sem uso de antihipertensivos. Os pacientes foram randomizados em dois grupos: GRUPO CONTROLE (GC) - orientados para TNF; e GRUPO ESTUDO (GE) - TNF e programa de atividade física supervisionada que consistia de três sessões semanais com uma hora cada. A duração do estudo foi de 6 meses com avaliações na inclusão no estudo (VI) e a cada três meses (V2 e V3). Toda a amostra foi submetida à avaliação clínica, eletrocardiograma (ECG), teste ergométrico, monitorização ambulatorial da pressão arterial (MAPA) e ecodopplercardiograma. Utilizamos o teste de Friedman para análise evolutiva das médias intragrupo e teste $T$ de Student para comparação de dados independentes entre os grupos.

Resultados. Foram randomizados 24 pacientes no GE (Cinco homens) e 21 no GC (Quatro homens), destes 22 completaram os seis meses de seguimento no GE e 13 no GC. Os valores médios da pressão sistólica encontrados na MAPA

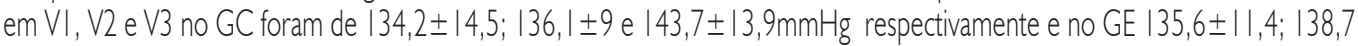
$\pm 12,2$ e $133,9 \pm 8,5 \mathrm{mmHg}$. Os valores médios da pressão diastólica no GC foram $78,7 \pm 5,82 ; 82,3 \pm 6,2$ e 83,3 $\pm 9,2 \mathrm{mmHg}$ e no GE $80,1 \pm 6,8 ; 81 \pm 6,6$ e $80,8 \pm 7,3 \mathrm{mmHg}$.
\end{abstract}

Conclusão. A atividade física supervisionada foi mais eficiente em manter o controle da pressão arterial em idosos com hipertensão estágio I (GE) quando comparada ao grupo controle.

UNITERMOS: Hipertensão. Atividade física. Idoso.

\section{INTRODUÇÃO}

No Brasil, a prevalência da hipertensão arterial $(H A)$ varia de $22,3 \%$ a $43,9 \%$ e os dados epidemiológicos nacionais e internacionais demonstram que a elevação da pressão arterial (PA) está intimamente relacionada ao processo de envelhecimento, principalmente os valores da pressão sistólica, ${ }^{1.7}$ influencia no aumento do risco cardiovascular, e portanto deve ser tratada 8,9 .

Com relação ao TNF, todas as diretrizes citam maior efetividade com as medidas de redução do peso, restrição de sódio, prática regular de atividade física e consumo moderado de álcool ${ }^{10-15}$.

Diversos estudos demonstram o papel da atividade física na redução da pressão arterial e da morbimortalidade cardiovascular ${ }^{16-23} \mathrm{e}$ vários são os mecanismos envolvidos no efeito hipotensor do treinamento físico, que se torna mais evidente a partir da décima semana de treinamento com pequenos ganhos adicionais subseqüentes ${ }^{24-27}$.

A atividade física deve ser avaliada e prescrita em termos de intensidade, frequêencia, duração, modo e progressão. A escolha do tipo de atividade física deverá ser orientada de acordo com as preferências individuais, respeitando as limitações impostas pela idade, como evitar o estresse ortopédico ${ }^{28}$. Os exercícios resistidos de intensidade leve (40\% a 60\% da carga voluntária máxima), com um número maior de repetições também parecem ter efeito benéfico na PA, além dos benefícios comprovados sobre o sistema osteomuscular, podem, portanto, ser prescritos para o hipertenso desde que estejam associados aos exercícios aeróbios ${ }^{29}$.

A ação dos exercícios aeróbios e resistidos nos níveis tensionais ainda são objetos de estudo em todo o mundo. Só este fato já justifica a proposta deste trabalho, que é de avaliar, na população de idosos com HA, os efeitos da atividade física programada e supervisionada (prescrita e acompanhada por profissionais habilitados) comparada com a atividade física convencional, tão amplamente divulgada pelas diretrizes médicas.

\section{MÉtodos}

O protocolo do estudo foi submetido e aprovado pelo Comitê de Ética em Pesquisa Humana e Animal do Hospital das Clínicas $(H C)$ da Universidade Federal de Goiás (UFG). O termo de consentimento livre e esclarecido foi obtido de todos os pacientes antes que qualquer procedimento relacionado ao estudo fosse executado. 
O tamanho da amostra calculado para uma variação de PAS de $4 \mathrm{mmHg}$, nível de significância mínimo de alfa $<0,05$ foi de 30 pacientes para o grupo estudo e 30 para o grupo controle.

Os critérios de inclusão foram pacientes maiores de 60 anos, PAD $\geq 90 \mathrm{mmHg}$ e $<100 \mathrm{mmHg}$ e/ou PAS $\geq 140 \mathrm{mmHg}$ e $<160 \mathrm{mmHg}$, sem uso de anti-hipertensivos há pelo menos duas semanas.

Os critérios de exclusão foram: evidência de lesões em órgãosalvo, hipertensão secundária, hipertensão malígna ou acelerada, creatinina $>2 \mathrm{mg} / \mathrm{dl}$ coronariopatia sintomática, arritmias graves, bloqueios atrioventriculares $2^{\circ}$ ou $3^{\circ}$ graus, insuficiência cardíaca congestiva, antecedente de acidente vascular cerebral (AVC), valvopatias significativas, doenças hepáticas (TGO e/ou TGP com valores duas vezes acima dos normais), história de alcoolismo ou uso de drogas ilícitas, elevação da PA durante o estudo para valores acima dos permitidos nos critérios de inclusão.

Para a inclusão no estudo, foram considerados os valores obtidos pela aferição da pressão casual, sendo adotada a média da pressão obtida em duas consultas independentes e subsequentes (média de três medidas em cada consulta). A pressão foi avaliada com esfigmomanômetro de coluna de mercúrio, com paciente na posição supina, após 10 minutos de repouso em ambiente tranqüilo, respeitando o intervalo de dois minutos entre cada aferição da $\mathrm{PA}^{30}$.

Todos os pacientes receberam orientação para o TNF conforme rotina do serviço oferecido pelo HC da UFG, que consta de dieta hipossódica com 4 a $7 \mathrm{~g}$ de $\mathrm{NaCl} / \mathrm{dia}$, restrição ao uso de bebidas alcoólicas, estímulo ao abandono do tabagismo, redução de peso (quando indicado), incentivo à ingestão de maior quantidade de fibras na alimentação e orientação para a prática de caminhadas diárias de pelo menos 30 minutos.

Os pacientes foram alocados aleatoriamente em dois grupos:

- Grupo C (Controle): Formado por pacientes que foram orientados para TNF.

- Grupo E (Estudo): Formado por pacientes que, além do TNF, foram incluídos em um programa de atividade física programada e supervisionada, sendo que nestes dias eram orientados a não realizar as caminhadas de 30 minutos referentes ao TNF.

As variáveis analisadas foram a medida da pressão arterial casual pelo pesquisador, a média da PA na MAPA nas 24 horas, e resposta pressórica durante o teste ergométrico.

De um total de 1.600 pacientes matriculados no serviço, foram analisados os prontuários dos 342 pacientes acima de 60 anos (21\%). Após seleção, baseada nos critérios de inclusão e exclusão, os pacientes foram encaminhados para a fase de retirada dos anti-hipertensivos durante duas semanas e aqueles que apresentaram hipertensão estágio I no final desta fase foram incluídos no presente estudo.

Apesar da revisão do prontuário de todos os pacientes matriculados e regularmente acompanhados na Liga de Hipertensão do HC-UFGO não conseguimos selecionar o n inicialmente proposto devido à dificuldade de adequação aos critérios de inclusão/exclusão.

Todos os pacientes foram submetidos, para a inclusão no estudo e nas visitas trimestrais, aos exames de ECG, ecodopplercardiograma, TE e MAPA. Realizamos também o cálculo do índice de massa corporal (IMC) pela fórmula: peso $(\mathrm{Kg}) \div$ altura².
A atividade física supervisionada foi realizada no Serviço de Reabilitação Cardíaca do HC da UFG, com orientação de equipe multidisciplinar composta de cardiologista, educador físico e fisioterapeuta. O programa constou de sessões com uma hora de duração, com periodicidade de três sessões por semana. Os pacientes foram submetidos à prática de atividades aeróbias em bicicleta e esteira rolante, tendo como meta a manutenção da freqüência cardíaca entre 60\% e $75 \%$ da freqüência cardíaca máxima (FCM) atingida no pico do esforço no TE. A atividade aeróbia foi realizada durante 30 minutos em cada sessão. Os outros 30 minutos foram complementados com exercícios de flexibilidade e atividades com pesos (exercícios resistidos) com cargas de 40\% a 60\% da repetição máxima (RM) em três séries de 10 repetições ${ }^{3 !}$.

Para a medida casual da pressão arterial foram adotadas as técnicas recomendadas pelas IV Diretrizes Brasileiras de Hipertensão Arterial $(2002)^{3}$.

A MAPA foi obtida por meio de monitor Spacelabs 9002 (método oscilométrico). Foram padronizadas medidas a cada 15 minutos no período de viǵlia (7 às 23 horas) e a cada 20 minutos no período de sono (23 às 7 horas), sendo que os dados foram considerados válidos quando a monitorização aconteceu por um período de 24 horas \pm 2 horas e que o percentual de sucesso de leituras foi superior a $80 \%$ das medidas realizadas ${ }^{32}$

O TE foi realizado em esteira rolante da marca Ecafix, seguindo o Protocolo de Bruce, com programa Dixtal para análise e registro de três derivações eletrocardiográficas simultâneas (CM5, aVF e V2). Realizou-se como rotina o eletrocardiograma em repouso, com 12 derivações, seguido ainda, na fase do pré-esforço, de ECG com três derivações na posição ortostática e aferição da pressão arterial (PA) nesta posição. A fase do esforço foi realizada em esteira rolante com aferição da PA e registro eletrocardiográfico a cada três minutos ou a critério médico. Na fase de recuperação, os registros foram realizados nos $1^{\circ}, 2^{\circ}$ e $4^{\circ}$ minutos. A distância percorrida foi avaliada em metros e o cálculo do $\mathrm{VO}^{2}$ máximo obtido indiretamente através de fórmulas empregadas para este fim no software Dixtal.

A interpretação do exame foi realizada sempre pelo mesmo cardiologista, seguindo as recomendações do Consenso Nacional de Ergometria ${ }^{33}$.

O ecodopplercardiograma foi realizado nos serviços de ecocardiografia do HC da UFG, e do Hospital do Coração de Goiás, sendo feitos pelo mesmo ecocardiografista.

Os pacientes foram acompanhados por um período de 24 semanas sendo que para a análise dos dados da pressão utilizamos os achados da visita de inclusão (VI), após I 2 semanas (V2) e no final do estudo (V3). Durante os seis meses de seguimento todos os pacientes (GE e GC) receberam placebo em substituição ao tratamento farmacológico anti-hipertensivo.

$\mathrm{Na}$ avaliação dos dados foram realizadas inicialmente comparações intragrupo, sendo utilizado para análise estatística o teste de Friedman (teste paramétrico, para dados pareados). Ao constatar diferenças significativas no teste de Friedman, realizou-se um segundo teste (T Student para dados pareados).

Foram a seguir feitas comparações entre os grupos e para a análise estatística foi utilizado o teste T Student. 
BARROSO WKS ET AL.

\section{Resultados}

Foram randomizados 24 pacientes para o GE e 2 I para o GC sendo que havia cinco pacientes do sexo masculino no GE ${ }^{4}$ no GC. Destes, 22 pacientes completaram os seis meses de seguimento no GE e I 3 no GC. A causa de interrupção do estudo foi a elevação da PA para valores iguais ou maiores que $160 \times 100 \mathrm{mmHg}$ (critério para retirada do paciente e reinício do tratamento farmacológico com drogas hipotensoras). Não ocorreram, em nenhum dos pacientes, complicações cardiovasculares ou eventos que fossem considerados como sérios.

A idade média foi de 66,5 \pm 4 anos (6I a 79) no GE e 70,8 \pm 6,3 anos (6 | a 77) no GC. ( $p>0,05)$.

As características basais das variáveis analisadas no GE e GC foram homogêneas para a grande maioria dos dados sendo encontradas diferenças significativas na fase inicial apenas para distância percorrida no teste ergométrico: GE $394 \mathrm{~m} \pm$ 135,4; GC 263,6 m $\pm 169,2$ ( $p=$ 0,030), VO2 máximo: GE 25,2 $\mathrm{mlO}^{2} / \mathrm{Kg} / \mathrm{min} \pm 7,7 ; \mathrm{GC} 19,7 \mathrm{mlO}^{2} / \mathrm{Kg} /$ $\min \pm 7,3(p=0,046)$, triglicérides: GE I50,9 mg/d $\pm 78,6 ; G C \mid 05,8$ $\pm 25,8$ ( $p=0,023)$, diâmetro do septo interventricular: GE $9,2 \mathrm{~mm}$ $\pm 1,71 ; G C 8,1 \mathrm{~mm} \pm 1, I(p=0,032)$, diâmetro do átrio esquerdo: GE 34,2 mm $\pm 4, I$; GC 31,7 mm $\pm 2, I(p=0,033)$.

$\mathrm{Na}$ avaliação intragrupo encontramos aumento significativo da distância percorrida de VI para V3 $(394, I m, 516,3 m-p=0,021)$ no GE e não significativa no GC $(263,5 \mathrm{~m}$ e $356,9 \mathrm{~m}$ em VI e V3 respectivamente). Os valores da pressão casual sofreram discreta diminuição no GE e discreta elevação no GC, sem, entretanto alcançar significância estatística em ambos os casos. Na análise pelo MAPA (Tabela I), observamos alterações apenas no GC, com elevação progressiva e significativa da PAS (I34,2 mmHg, I36,I mmHg e $143,7 \mathrm{mmHg}$ de $\mathrm{VI}$ a V3, $p=0,039)$ e da PAD $(78,7 \mathrm{mmHg}, 82,3 \mathrm{mmHg}$ e $83,3 \mathrm{mmHg}$ de $V I$ a $V 3 ; p=0,05$.

Na comparação entre grupos encontramos os resultados descritos na Tabela 2. Cumpre observar as mudanças nas médias da PAS e PAD (Figuras I e 2), com tendência a diminuição da PA no GE e a aumento no GC, no entanto, só ocorreu significância estatística na comparação da PAD em V3 ( $p=0,0 \mid 2)$. Na avaliação pela MAPA, encontramos as mesmas tendências de evoluçãa, sempre com diminuição dos valores da pressão no GE e elevação no GC, sem alcançar significância estatística em qualquer um dos casos.

Ao teste ergométrico, os valores da distância percorrida e o $\mathrm{VO}^{2}$ máximo já apresentavam diferenças na randomização, denotando melhor condicionamento físico no GE, e essa diferença se manteve em V2 e V3.

Quando analisamos apenas as pacientes do sexo feminino os valores encontrados foram semelhantes ao da amostra total.

\section{Discussão}

A hipertensão arterial é uma das patologias de maior prevalência na população adulta e principalmente nos idosos. Está associada ao aumento na morbidade-mortalidade por todas as causas e cardiovascular e os programas de exercício estão associados à prevenção do desenvolvimento de hipertensão e também à redução da PA, tanto em normotensos quanto em hipertensos.

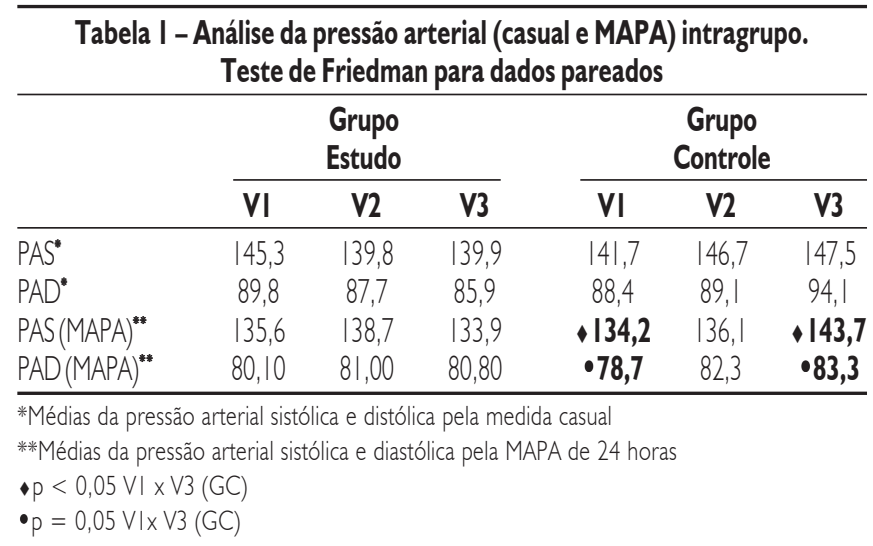

A proposta deste estudo foi avaliar comparativamente, em idosos portadores de hipertensão estágio I, a atividade física programada e supervisionada (Reabilitação cardíaca) à atividade física realizada de forma convencional pelo paciente.

Em relação à segurança da atividade física proposta e praticada, não observamos nos dois grupos a ocorrência de eventos adversos relacionados aos exercícios nos seis meses de seguimento. Vale ressaltar que a atividade física proposta era de intensidade leve a moderada e prescrita após avaliação clínica e exames complementares, respeitando as limitações e o condicionamento físico de cada indivíduo.

Com relação à randomização do estudo, atingiu-se $80 \%$ da meta proposta para a amostra no GE e $70 \%$ no GC. A maior dificuldade para a randomização foi encontrar indivíduos hipertensos na faixa etária do protocolo que pudessem ficar seis meses em uso de placebo, dentro de normas éticas. Observou-se ainda um predomínio do sexo feminino na amostra estudada (79\% em GE e $80 \%$ em GC), o que é compatível com a população acompanhada na Liga de Hipertensão Arterial da UFG. Neste aspecto, ao realizarmos a análise estatística utilizando apenas os dados do sexo feminino, os resultados foram semelhantes.

As características basais de GE e GC foram homogêneas para praticamente todas as variáveis analisadas, entretanto ocorreram diferenças significativas com relação aos parâmetros do condicionamento físico no teste ergométrico, no qual se encontrou uma população melhor condicionada no Grupo Estudo, demonstrada pela distância percorrida no TEI (394, I m \pm 135,4 vs 263,5 $\mathrm{m} \pm 169,2$ em GE e GC respectivamente; $p=0,030)$ e pelo $\mathrm{VO}^{2}\left(25,2 \mathrm{mlO}^{2} /\right.$ $\mathrm{Kg} / \mathrm{min} \pm 7,7 \mathrm{vs} 19,7 \mathrm{mlO} / \mathrm{Kg} / \mathrm{min} \pm 7,3 \mathrm{em}$ GE e GC; $p=0,046)$. Também foram encontradas diferenças significativas nos dados basais obtidos no ecodopplercardiograma para avaliação do diâmetro do septo interventricular ( $9,2 \mathrm{~mm} \pm 1,7$ em GE e 8, I mm \pm I , I em GC; $p=0,032)$ e do átrio esquerdo $(34,2 \mathrm{~mm} \pm 4$ e $31,7 \mathrm{~mm} \pm 2$, I em GE e GC respectivamente; $p=0,033$ ) embora estivessem todo o tempo dentro dos parâmetros de normalidade. Quanto a PA basal obtida pela medida casual e pela MAPA, observou-se valores maiores no Grupo Estudo, tanto para a PAS quanto para PAD, mas sem significância estatística. Cumpre ressaltar que estas diferenças apontando para um GE com melhor condicionamento físico podem ter provocado vieses na análise evolutiva dos grupos, por outro lado também devemos considerar os maiores valores basais da pressão 


\begin{tabular}{|c|c|c|c|c|c|}
\hline \multicolumn{6}{|c|}{$\begin{array}{c}\text { Tabela } 2 \text { - Análise comparativa de GE x GC (Entre grupos) nas } \\
\text { visitas I, } 2 \text { e 3. Teste T para dados independentes }\end{array}$} \\
\hline Variável & & & $D-P$ & $T$ & $\mathbf{P}$ \\
\hline$\overline{\mid M C V I}$ & $\begin{array}{l}\text { GE: } \\
\text { GC: }\end{array}$ & $\begin{array}{l}27,3 \\
26,2\end{array}$ & $\begin{array}{l}3,7 \\
5,7\end{array}$ & 0,699 & 0,489 \\
\hline IMCV2 & $\begin{array}{l}\text { GE: } \\
\text { GC: }\end{array}$ & $\begin{array}{l}27,5 \\
26,3\end{array}$ & $\begin{array}{l}3,9 \\
5,4\end{array}$ & 0,775 & 0,444 \\
\hline IMCV3 & $\begin{array}{l}\text { GE: } \\
\text { GC: }\end{array}$ & $\begin{array}{l}27,7 \\
27,7\end{array}$ & $\begin{array}{l}4,2 \\
5,3\end{array}$ & 0,032 & 0,975 \\
\hline PASVI & $\begin{array}{l}\text { GE: } \\
\text { GC: }\end{array}$ & $\begin{array}{l}145,3 \\
|4|, 7\end{array}$ & $\begin{array}{l}8,4 \\
8,1\end{array}$ & 1,238 & 0,225 \\
\hline PAS V2 & $\begin{array}{l}\text { GE: } \\
\text { GC: }\end{array}$ & $\begin{array}{l}139,8 \\
146,7\end{array}$ & $\begin{array}{l}14,3 \\
15,3\end{array}$ & |,347 & 0,187 \\
\hline PAS V3 & $\begin{array}{l}\text { GE: } \\
\text { GC: }\end{array}$ & $\begin{array}{l}139,9 \\
147,5\end{array}$ & $\begin{array}{l}13,8 \\
16,6\end{array}$ & 1,278 & 0,213 \\
\hline PAD VI & $\begin{array}{l}\text { GE: } \\
\text { GC: }\end{array}$ & $\begin{array}{l}89,9 \\
88,4\end{array}$ & $\begin{array}{l}5,9 \\
7,9\end{array}$ & 0,631 & 0,533 \\
\hline PAD V2 & $\begin{array}{l}\text { GE: } \\
\text { GC: }\end{array}$ & $\begin{array}{l}87,7 \\
89,1\end{array}$ & $\begin{array}{l}7,5 \\
8,1\end{array}$ & 0,511 & 0,613 \\
\hline *PADV3 & $\begin{array}{l}\text { GE: } \\
\text { GC: }\end{array}$ & $\begin{array}{l}85,9 \\
94,1\end{array}$ & $\begin{array}{c}5,6 \\
10,5\end{array}$ & 2,711 & $0,012 *$ \\
\hline PAST VI & $\begin{array}{l}\text { GE: } \\
\text { GC: }\end{array}$ & $\begin{array}{l}203,8 \\
193,8\end{array}$ & $\begin{array}{c}20,4 \\
21\end{array}$ & 1,369 & 0,180 \\
\hline PASTVZ & $\begin{array}{l}\text { GE: } \\
\text { GC: }\end{array}$ & $\begin{array}{l}210,7 \\
211,8\end{array}$ & $\begin{array}{l}18,2 \\
19,9\end{array}$ & 0,145 & 0,886 \\
\hline PAST V3 & $\begin{array}{l}\text { GE: } \\
\text { GC: }\end{array}$ & $\begin{array}{c}216 \\
201,2\end{array}$ & $\begin{array}{l}15,5 \\
22,3\end{array}$ & 1,866 & 0,076 \\
\hline PADT VI & $\begin{array}{l}\text { GE: } \\
\text { GC: }\end{array}$ & $\begin{array}{l}104,8 \\
101,5\end{array}$ & $\begin{array}{l}14,7 \\
\mid 7,7\end{array}$ & 0,574 & 0,570 \\
\hline PADT V2 & $\begin{array}{l}\text { GE: } \\
\text { GC: }\end{array}$ & $\begin{array}{l}100,7 \\
107,3\end{array}$ & $\begin{array}{l}14,4 \\
15,5\end{array}$ & 1,092 & 0,286 \\
\hline PADT V3 & $\begin{array}{l}\text { GE: } \\
\text { GC: }\end{array}$ & $\begin{array}{l}107,3 \\
103,7\end{array}$ & $\begin{array}{c}16,2 \\
13\end{array}$ & 0,537 & 0,597 \\
\hline *Dist VI & $\begin{array}{l}\text { GE: } \\
\text { GC: }\end{array}$ & $\begin{array}{l}394,1 \\
263,5\end{array}$ & $\begin{array}{l}135,4 \\
169,2\end{array}$ & 2,292 & $0,030 *$ \\
\hline *Dist V2 & $\begin{array}{l}\text { GE: } \\
\text { GC: }\end{array}$ & $\begin{array}{l}514,7 \\
277,7\end{array}$ & $\begin{array}{l}219,4 \\
185,7\end{array}$ & 2,610 & $0,017 *$ \\
\hline *Dist V3 & $\begin{array}{l}\text { GE: } \\
\text { GC: }\end{array}$ & $\begin{array}{l}516,3 \\
356,9\end{array}$ & $\begin{array}{l}149, \mid \\
144,6\end{array}$ & 2,329 & $0,032 *$ \\
\hline *VO $2 \max \mathrm{VI}$ & $\begin{array}{l}\text { GE: } \\
\text { GC: }\end{array}$ & $\begin{array}{l}25,2 \\
19,7\end{array}$ & $\begin{array}{l}7,7 \\
7,3\end{array}$ & 2,081 & $0,046 *$ \\
\hline *VO 2 max V2 & $\begin{array}{l}\text { GE: } \\
\text { GC: }\end{array}$ & $\begin{array}{l}29,2 \\
18,7\end{array}$ & $\begin{array}{l}9,7 \\
7,8\end{array}$ & 2,936 & 0,007 * \\
\hline *VO2max V3 & $\begin{array}{l}\text { GE: } \\
\text { GC: }\end{array}$ & $\begin{array}{l}29,6 \\
21,2\end{array}$ & $\begin{array}{l}6,4 \\
6,2\end{array}$ & 2,953 & $0,008 *$ \\
\hline PAMs VI & $\begin{array}{l}\text { GE: } \\
\text { GC: }\end{array}$ & $\begin{array}{l}135,6 \\
134,2\end{array}$ & $\begin{array}{l}11,4 \\
14,5\end{array}$ & 0,290 & 0,774 \\
\hline PAMs V2 & $\begin{array}{l}\text { GE: } \\
\text { GC: }\end{array}$ & $\begin{array}{l}\mid 38,7 \\
|36,|\end{array}$ & $\begin{array}{c}12,2 \\
9\end{array}$ & 0,574 & 0,571 \\
\hline PAMs V3 & $\begin{array}{l}\text { GE: } \\
\text { GC: }\end{array}$ & $\begin{array}{l}133,9 \\
143,7\end{array}$ & $\begin{array}{l}8,5 \\
13,9\end{array}$ & 2,053 & 0,053 \\
\hline PAMdVI & $\begin{array}{l}\text { GE: } \\
\text { GC: }\end{array}$ & $\begin{array}{l}80,1 \\
78,7\end{array}$ & $\begin{array}{l}6,8 \\
5,8\end{array}$ & 0,574 & 0,570 \\
\hline PAMd V2 & $\begin{array}{l}\text { GE: } \\
\text { GC: }\end{array}$ & $\begin{array}{c}81 \\
82,3\end{array}$ & $\begin{array}{l}6,6 \\
6,2\end{array}$ & 0,505 & 0,618 \\
\hline PAMd V3 & $\begin{array}{l}\text { GE: } \\
\text { GC: }\end{array}$ & $\begin{array}{l}80,8 \\
83,3 \\
\end{array}$ & $\begin{array}{l}7,3 \\
9,2 \\
\end{array}$ & 0,682 & 0,503 \\
\hline
\end{tabular}

* $p<0,05$ na análise entre-grupos

$I M C=$ índice de massa corporal; PAS = pressão arterial sistólica $(\mathrm{mmHg}) ; P A D=$ pressão arterial diastólica $(\mathrm{mmHg}) ;$ PAST = pressão arterial sistólica no pico do esforço do teste ergométrico; PADT = pressão arterial diastólica no pico do esforço do teste ergométrico; DP = duplo produto; Dist. = distância percorrida em metros no teste ergométrico; PAMs = pressão arterial sistólica na MAPA (Média das 24hs); PAMd = pressão arterial diastólica na MAPA (Média das 24hs).
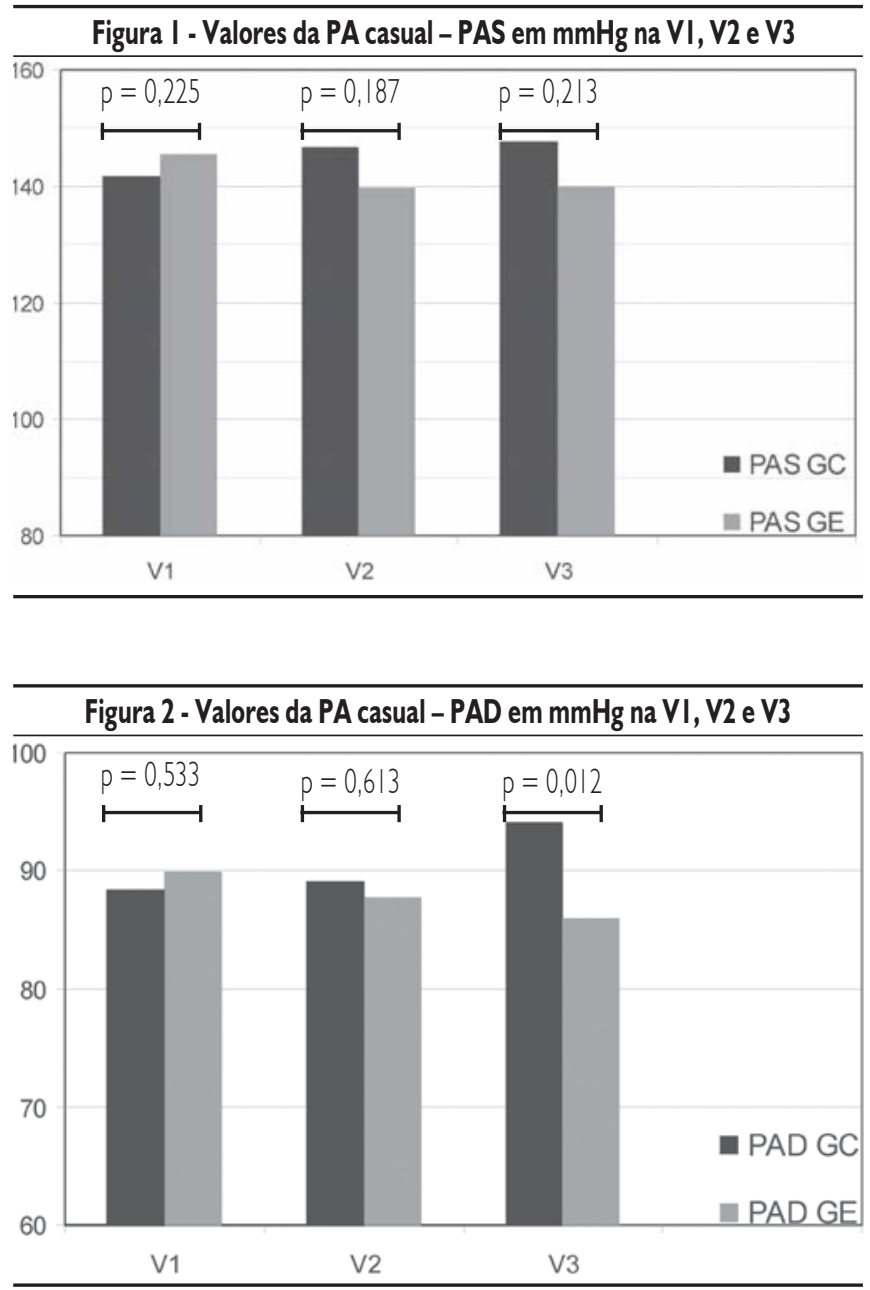

arterial no GE uma vez que estes atuariam de forma desfavorável nas reduções esperadas da PA.

Os grupos se apresentaram com sobrepeso na randomização (IMC $=27,4 \pm 3,7$ e 26,2 $\pm 5,7$ em GE e GC). Este dado coincide com tantos outros da literatura, que encontraram forte associação entre sobrepeso/obesidade e hipertensão ${ }^{34,35}$. Nesta amostra não ocorreu diminuição do IMC após os seis meses e estes achados já eram esperados pelo nosso grupo, com base na experiência prévia com os pacientes da Liga de Hipertensão onde temos observado grandes dificuldades em atingir e manter as metas de redução de peso nos pacientes hipertensos, mesmo naqueles que aderem às orientações para a prática da atividade física.

Com relação à análise evolutiva intragrupo no GE observa-se na medida casual da PA uma discreta diminuição dos valores tanto da PAS quanto da PAD, principalmente na V3, sem, entretanto atingir significância na análise estatística (Tabela I). A nosso ver, estes números demonstram que a atividade física supervisionada foi capaz de manter os níveis pressóricos semelhantes aos valores basais mesmo após 6 meses sem uso de medicamentos hipotensores. Com relação às medidas da PA na MAPA, observou-se também uma discreta diminuição da PAS e da PAD na V3, novamente sem significância estatística. 
BARROSO WKS ET AL.

A literatura descreve uma redução discreta ou neutra da PA, principalmente após a $10^{\circ}$ semana de atividade física regularl 1,24 e também descreve uma menor redução nos valores tensionais quando avaliados com a MAPA, porém este último método se mostra mais eficaz na predição do risco cardiovascular a longo prazo ${ }^{36-42}$.

O teste ergométrico nos indivíduos do GE não mostrou diferenças na PAS e na PAD aferidas no pico do esforço, entretanto ocorreu um aumento significativo na distância percorrida de VI para V3, ou seja, os pacientes conseguiram caminhar um tempo maior na esteira mantendo níveis tensionais semelhantes aos do início do estudo, podendo denotar, a nosso ver, melhora do controle pressórico frente ao esforço físico concomitante com a melhora do condicionamento.

Com relação à análise evolutiva intragrupo no GC observamos aumento da PA na medida casual embora sem significância estatística. $\mathrm{Na}$ avaliação pela MAPA, a mesma ocorrência de aumento da PA é observada com $p<0,05$ na PAS e $p=0,05$ na PAD (análise de VI vsV3). Tais dados demonstram que neste grupo a atividade física orientada da forma convencional não foi capaz de manter os níveis tensionais basais. Há que se considerar ainda a possibilidade de baixa adesão às orientações para a prática da atividade física visto que ao avaliarmos os dados de condicionamento físico (distância e $\mathrm{VO}^{2}$ max) encontramos valores menores nos seis meses de seguimento.

O teste ergométrico nos indivíduos do GC, assim como no GE, não mostrou diferenças na PAS e PAD aferidas no pico do esforço. No GC, entretanto, não ocorreu aumento da distância percorrida, que pode se relacionar a suposta baixa adesão às orientações para a atividade física, ou ainda conseqüência do menor condicionamento físico basal.

Finalmente, foram realizadas análises comparativas entre GE e GC (Tabela 2), nesta avaliação apenas a PAD na V3 mostrou diferenças significativas entre os grupos $(\mathrm{GE}=85,9 \mathrm{mmHg} \pm 5,6$ e $\mathrm{GC}=94$, I $\mathrm{mmHg} \pm 10,5 ; p=0,0 \mid 2$ ). As pressões avaliadas pela MAPA em todos os momentos não apresentaram diferenças significativas.

$\mathrm{Na}$ análise do abandono do estudo, nota-se que no GC oito indivíduos (38\%) contra dois (8\%) no GE não completaram os seis meses de seguimento devido à elevação da PA para valores maiores que os permitidos no protocolo. A grande taxa de abandono no Grupo Controle constitui uma limitação importante para a análise dos resultados e pode ser atribuída a uma maior adesão ao programa de atividade física com melhor controle pressórico no grupo de atividade física supervisionada; entretanto esta hipótese não foi testada e, portanto, não pode ser confirmada.

\section{Conclusão}

Concluímos que programas de atividade física supervisionada podem ser úteis na implementação do tratamento não-farmacológico da HA e que, nesta amostra, a associação dos exercícios aeróbios com os resistidos e de flexibilidade se mostrou segura, apontando para mais uma opção na prescrição do exercício nos indivíduos com este perfil.

A atividade física supervisionada (GE) foi mais eficiente em manter os níveis tensionais basais em idosos hipertensos sob tratamento não-farmacológico quando comparada à atividade física orientada de forma convencional (GC), demonstrando inclusive redução significativa da PAD na medida casual após seis meses de seguimento.
É possível que uma maior adesão ao exercício no GE tenha sido responsável por parte dos resultados encontrados.

Estas hipóteses devem ser testadas em um número maior de pacientes para que conclusões mais contundentes possam ser assumidas.

\section{Conflito de interesse: não há}

\section{SUMMARY}

The INFLUENCE OF PROGRAMMED PHYSICAL ACTIVITY ON BLOOD PRESSURE OF HYPERTENSIVE ELDERLY PATIENTS ON NONPHARMACOLOGICAL TREATMENT

OBIECTNE. Assess the influence of physicalactivity on the blood pressure of hypertensive elderly patients on non-pharmacological treatment (NPT).

METHODS. The authors studied men and women, over 60 years, with stage I hypertension who were not using antihypertensive medication. These patients had been randomly allocated to one of two groups: Control Group (CG)-orientedtoNPT; and Study Group(SG)-NPT andaprogram that included supervised physical activity consisting of I-hour sessions, 3 times a week. Duration of the study was 6 months. Screening and followup tests every 3 months, at Visits 1,2 and 3 included a clinical evaluation, ECG, a treadmill stress test, Ambulatory Blood Pressure Measurement (ABPM) and an Echocardiogram. The authors used the Friedman test for the evolutionary analysis of the intra-groups means and Student's ttest to compare independent data between groups.

RESULTS. Twenty-four patients were randomized in the SG (5 men) and 21 in the CG (4 men). The 6-month follow-up period was completed by 22 patients in the SG and 13 in the $C G$. Blood pressures found by the ABPM in VI, V2 and V 3 were $134.2 \pm 14.5,136.1 \pm 9$ and $143.7 \pm 13.9 \mathrm{mmHg}$ for Systolic Blood Pressure in the CG; and 135.6 \pm 1 I.4, 138.7 \pm 12.2 and $133.9 \pm 8.5 \mathrm{mmHg}$ in the SG. For Diastolic Blood Pressure, values in the CG were $78.7 \pm 5.8,82.3 \pm 6.2$ and $83.3 \pm 9.2 \mathrm{mmHg}$; and in the $S G$, $80.1 \pm 6.8,81 \pm 6.6$ and $80.8 \pm 7.3 \mathrm{mmHg}$.

CONCLUSION. Programmed and supervised physical activity was more efficient for maintaining adequate blood pressure control in these elderly with stage I hypertension in the study group, when compared to the control group. [Rev Assoc Med Bras 2008; 54(4): 328-33]

KEY wORDs: Hypertension. Physical activity. Elderly.

\section{REFERÊNCIAS}

I. World Health Organization. International Society of Hypertension Guidelines for the Management of Hypertension. J Hypertens. 1999; 17: | 51-83.

2. The Seventh Report of The Joint National Committee on Prevention, Detection, Evaluation, and Treatment of High Blood Pressure. The JNC 7 Report. JAMA. 2003;289:2560-72.

3. IV Diretrizes Brasileiras de Hipertensão Arterial. Hipertensão. 2002;5: I 23-63.

4. Berlowitz DR, Ash AS, Hickey EC, Friedman NRH, Glockman M, Kader $B$, et al. Inadequate management of blood pressure in a hypertensive population. N Engl J Med. 1998;339: 1957-63.

5. Jardim PCBV, Barroso WKS. estudo da prevalência da hipertensão arterial em uma capital brasileira. Arq Bras Cardiol. 2002;79(Supl $3): 43$.

6. Verdecchia P. Angeli F. Natural history of hypertension subtypes. Circulation. 2005; I 1 1: 1094-6. 
7. Franklin SS, Pio JR, Wong ND, Larson MG, Leip EP, Vasan RS, et al. Predictors of new-onset diastolic and systolic hypertension. The Framinghan Heart Study. Circulation. 2005; | I I : I I 2 I-7.

8. Gennari FJ. Hypertension in the elderly: management issues. Coron Artery Dis. 1998;9:719-24

9. Gueyffier F. Antihypertensive drugs in very old people: a subgroup metaanalysis of randomised controlled trials. Lancet. 1999;356:793-6.

10. Norman RC, Campbell MD, Ellen B, Gregory T, Elinor W, Jean C, et al. Lifestyle changes to prevent and control hypertension: Do they work? CMAJ. I999; | 60: | 341-3.

I I. Kokkinos PF, Narayan P, Papademetriou V. Exercise as a hypertension therapy. Cardiol Clin. 200 I; I 9:507- I 5.

12. NHANES I: Epidemiologic Follow up Study, 1992 Vital and Tracing Status. Hyattsville: US Department of Health and Human Services, Public Health Service, Center for Disease Control and Prevention. National Center for Health Statistics; 1996.

13. Jardim PCBV, Monego ET, Reis MA. Potássio, cálcio, magnésio e hipertensão arterial. Rev Bras Hipertens. 2004; I I : I09- I I.

14. Alderman MH, Cohen H, Madhavan S. Dietary Sodium Intake and Mortality: The National Health and Nutrition Examination Survey (NHANES I). Lancet. 1998;351:781-5.

15. Barroso WKS, Amaral GF. Álcool, hipertensão arterial e doença cardiovascular. Rev Bras Hipertens. 2004; I I : I I 2-4..

16. Blumenthal J, Sherwood A, Gullete ECD, Babyak M, Waugh R, Georgiades A, et al. Exercise and weight loss reduce blood pressure in men and women with mild hypertension. Arch Intern Med. 2000; 160: 1947-58.

17. Coope JR, Warrender TS. Randomised trial on treatment of hypertension in elderly patients in primary care. Br Med J. |986;293: I |45-5 I.

18. Larson EB, Edward K. Benefits of exercise for older adults. Clin Geriatr Med. 1992;8:35-47.

19. Ketelhut RG, Franz IW, Scholze J. Regular exercise as an effective approach in antihypertensive therapy. Med Sci Sports Exerc. 2004:36:4-8

20. Barroso WKS. Benefícios da atividade física na hipertensão arterial e orientações práticas. Rev Bras Hipertens. 2002; I I : | | 5-6.

21. Evenson KR, Stevens J, Thomas R, Cai J. Effect of cardiorespiratory fitness on mortality among hypertensive and normotensive women and men. Epidemiology. 2004; 1 5:565-72.

22. Barnes DE, Yaffe K, Satariano WA, Tager IB. A longitudinal study of cardiorespiratory fitness and cognitive function in healthy older adults. J Am Geriatr Soc. 2003;51:459-65.

23. Sandhya S, Jerry OC. Blood pressure in the elderly: an observation in octagenaries. J Clin Hypertens. 2000;2:263-6.

24. Pescatello LS, Franklin BA, Fagard R, Farquhar WA, Kelly GA, Ray CA, American College of Sports Medicine. Exercise and hypertension. Med Sci Sports Exerc. 2004; 36:533-53.

25. Lesniak KT, Dubbert PM. Exercise and hypertension. Curr Opini Cardiol. 2001; | 6:356-9.

26. Christmas C, Andersen RA. Exercise and older patients: guidelines for clinician. J Am Geriatr Soc. 2000;48:3 I 8-24.
27. Black HR, Cushman WC. Hypertension in the elderly. Cardiol Clin. 1999; 17:79-92.

28. Fletcher GF, Balady GJ, Amsterdam EA, Chaitman B, Eckel R, Froelicher $V F$, et al. Exercise standards for testing and training. A Statement for Healthcare Professionals From American Heart Association. Circulation. 200।; 104: 1694-40.

29. Pollock ML, Franklin BA, Balady GA, Chaitman BL, Fleg JL, Fletcher B, et al. Resistance exercise in individuals with and without cardiovascular disease. Circulation. 2000; 101:828-33.

30. Souza ALL. Manual de técnicas: medidas da pressão arterial e avaliação nutricional. Goiânia: CEGRAF; 1996.

31. I Consenso Nacional de Reabilitação Cardiovascular. Arq Bras Cardiol. |997;69:267-9|.

32. IV Diretriz Para o uso da Monitorização Ambulatorial da Pressão Arterial. Arq Bras Cardiol. 2005;85(Supl 2).

33. Consenso Nacional de Ergometria. Arq Bras Cardiol. 1995;65: I 89-92.

34. Hu G, Barengo C, Tuomilehto J, Lakka TA, Nissinen A, Jousilahti P. Relationship of physical activity and body mass index to the risk of hypertension: a prospective study in Finland. Hypertension. 2004:43:25-30.

35. Yanovski SZ, Yanovski JA. Obesity. N Engl J Med. 2002;346:59I-2.

36. Pickering TG, Phil D, Devereaux RB. Ambulatory monitoring of blood pressure as a predictor of cardiovascular risk. Am. Heart. J. | 987; | | 4:925-8.

37. Pickering TG, Hall JE, Appel LJ, Falkner BE, Graves SJ, Hill MN, et al. Recommendations for blood pressure measurement in humans and experimental animals. Hypertension. 2005;45: |42-61.

38. Shimada K, Kawamoto A, Matsubayashi K, Ozawa T. Silent cerebrovascular disease in the elderly: correlation with ambulatory blood pressure. Hipertension. 1990; 16 692-99.

39. Myers MG. Ambulatory blood pressure monitoring for routine clinical practice. Hypertension. 2005;45:483-4.

40. Ohkubo T, Imai Y, Tsuji I, Nagai K, Ito S, Satoh H, Hisamichi S. Reference values for 24-hour ambulatory blood pressure monitoring based on a prognostic criterion: The Ohasama Study. Hypertension. 1998;32:255-9.

4I. Clement DL, De Buyzere ML, De Bacquer DA, Duprez DA, Fagard $\mathrm{RH}$, Missault LH, et al. Prognostic value of ambulatory blood-pressure recordings in patients with treated hypertension. For the office versus ambulatory pressure study investigators. $N$ Engl J Med. 2003;348:2407- I 5.

42. Kikuia M, Okhubo T, Asayama K, Metoki H, Obara T, Saito S, et al. Ambulatory blood pressure and 10 -year risk of cardiovascular and noncardiovascular mortality. The Ohasama Study. Hypertension. 2005;45:240-5.

Artigo recebido: 06/08/07

Aceito para publicação: 01/04/08 